\title{
Nonequilibrium Spin-transfer Torque in SFNFS Junctions
}

\author{
Erhai Zhao and J. A. Sauls \\ Department of Physics and Astronomy, Northwestern University, Evanston, IL 60208, USA
}

\begin{abstract}
We report theoretical results for the nonequilibrium spin current and spin-transfer torque in voltage-biased SFNFS Josephson structures. The subharmonic gap structures and high voltage asymptotic behaviors of the dc and ac components of the spin current are analyzed and related to the spin-dependent inelastic scattering of quasiparticles at both $\mathrm{F}$ layers.
\end{abstract}

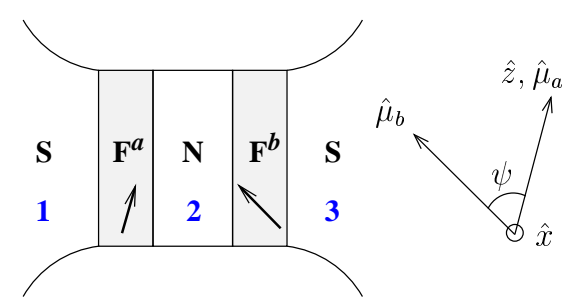

FIGURE 1. Schematic of SFNFS junction. The magnetization $\vec{\mu}_{a}$ is along the $z$ axis (the quantization axis for spin), while $\vec{\mu}_{b}$ is at polar angle $\psi$ in the $y z$ plane. The $x$ axis is along $\vec{\mu}_{a} \times \vec{\mu}_{b}$. Notice our choice of axis differs from Ref. [6].

Spin-polarized current passing through a ferromagnet can transfer spin angular momentum to the ferromagnet and exert a torque on the magnetic moment $[1,2]$. This mechanism offers unique opportunities to manipulate the magnetic state of nanomagnets. Experimentally spin-transfer torque driven magnetization precession and magnetization reversal have been observed in magnetic multilayers [3, 4]. A well studied multilayer system is the magnetic nanopillar which consists of a ferromagnetnormal metal-ferromagnet (FNF) trilayer connected to normal metal electrodes. The typical thickness of each layer is several $\mathrm{nm}$, and the diameter of the pillar is of the order of $50 \mathrm{~nm}$ [4]. When sandwiched between superconducting electrodes, the FNF trilayer can mediate finite Josephson coupling to form a SFNFS Josephson junction [5]. In such junctions, scattering of quasiparticle at the magnetic interfaces is sensitive to the phase shift in each F layer as well as the condensate phase difference $\phi$ across the junction. This indicates the spin momentum transfer between the quasiparticles and ferromagnets can be tuned by varying $\phi$. Waintal and Brouwer calculated the phase-sensitive equilibrium torque in SFNFS junctions [6]. They also showed the nonequilibrium torque in NFNFS junctions acquires novel features, e.g. it can favor perpendicular configuration of the two moments [7].
Here we investigate the nonequilibrium spin-transfer torque in SFNFS point contacts under bias voltage $V$. The setup and the coordinate system are shown in Fig. 1. The two F layers are labelled by indices $a$ and $b$, respectively. The $\mathrm{N}$ spacer is assumed to be transparent and in the clean limit. For simplicity we model each F layer as a delta function barrier with spin-dependent transmission probability $D_{\uparrow} \neq D_{\downarrow}$, so the spin mixing angle [8] $\vartheta=\arcsin \sqrt{D_{\uparrow}}-\arcsin \sqrt{D_{\downarrow}}$. The scattering matrix of $\mathrm{F}^{a}$ and $\mathrm{F}^{b}$ are related by spin rotation of angle $\psi$. Since the condensate phase difference evolves at Josephson frequency $\omega_{J}=2 \mathrm{eV} / \hbar$, the spin current in each region, $\mathbf{I}_{i}$ $(i=1,2,3)$, also oscillates with time. For example,

$$
\mathbf{I}_{2}(t)=\mathbf{I}_{0}+\sum_{k=1}^{\infty}\left[\mathbf{I}_{k, c} \cos \left(k \omega_{J} t\right)+\mathbf{I}_{k, s} \sin \left(k \omega_{J} t\right)\right] .
$$

We use the quasiclassical theory of superconductivity [9] and solve numerically the transport equations for the Green's functions in each region which obey proper boundary conditions at interface $\mathrm{F}^{a}$ and $\mathrm{F}^{b}$ [8]. The spin currents are then determined from the local Keldysh Green's functions. Within this approach the proximity effect and the multiple Andreev reflection (MAR) at both interfaces are fully taken into account.

The spin current in the $\mathrm{N}$ layer, measured in unit of $N_{f} v_{f} \Delta A \hbar / 2$ where $A$ is the contact cross sectional area and $\Delta$ is the superconducting gap, is shown in Fig. 2 for $\psi=\pi / 2$ and zero temperature. As in the case of nanopillars with normal metal electrodes, the dc spin current flow is a consequence of spin filtering at the $\mathrm{F}$ layers. Its voltage dependence, however, is nonlinear and possesses subharmonic gap structures due to the onset and resonances of MAR processes. At $V=0$, the dc spin current vector is along $x$ direction, which tends to cause the moments to precess around each other [6]. As $V$ is increased, the magnitude of dc currents along $y$ and $z$ direction, which tend to bring the moments towards or away from each other, grow with $V$. The 


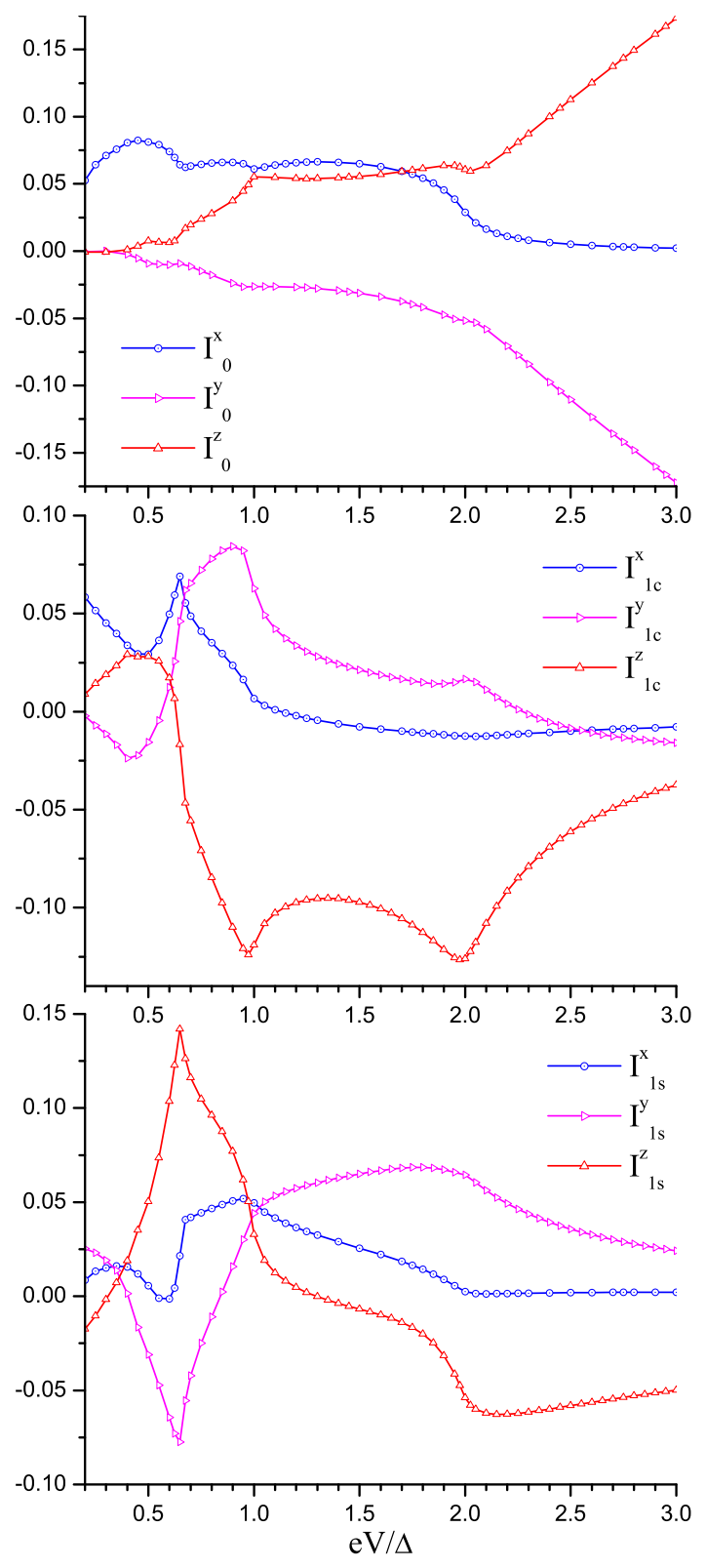

FIGURE 2. Dc (top panel), cosine (middle panel) and sine (bottom panel) part of the first Fourier components of the spin current in the $\mathrm{N}$ layer. Each panel features the spin current along $x, y$, and $z$ direction. $D_{\uparrow}=0.81, D_{\downarrow}=0.64, \psi=\pi / 2$.

ac spin current originates from the interference between MAR processes of different order. Its magnitude changes rapidly at voltages below $2 \Delta$ and decays to zero at high voltages.

The spin-transfer torque on $\mathrm{F}^{a}$ is given by $\vec{\tau}_{a}(t)=$ $\mathbf{I}_{1}(t)-\mathbf{I}_{2}(t)$, and the torque on $\mathrm{F}^{b}$ is given by $\vec{\tau}_{b}(t)=$ $\mathbf{I}_{2}(t)-\mathbf{I}_{3}(t)$. It is convenient to expand $\vec{\tau}_{a / b}(t)$ in Fourier series similar to Eq. (1). Fig. 3 shows the $k=0$ and $k=1$

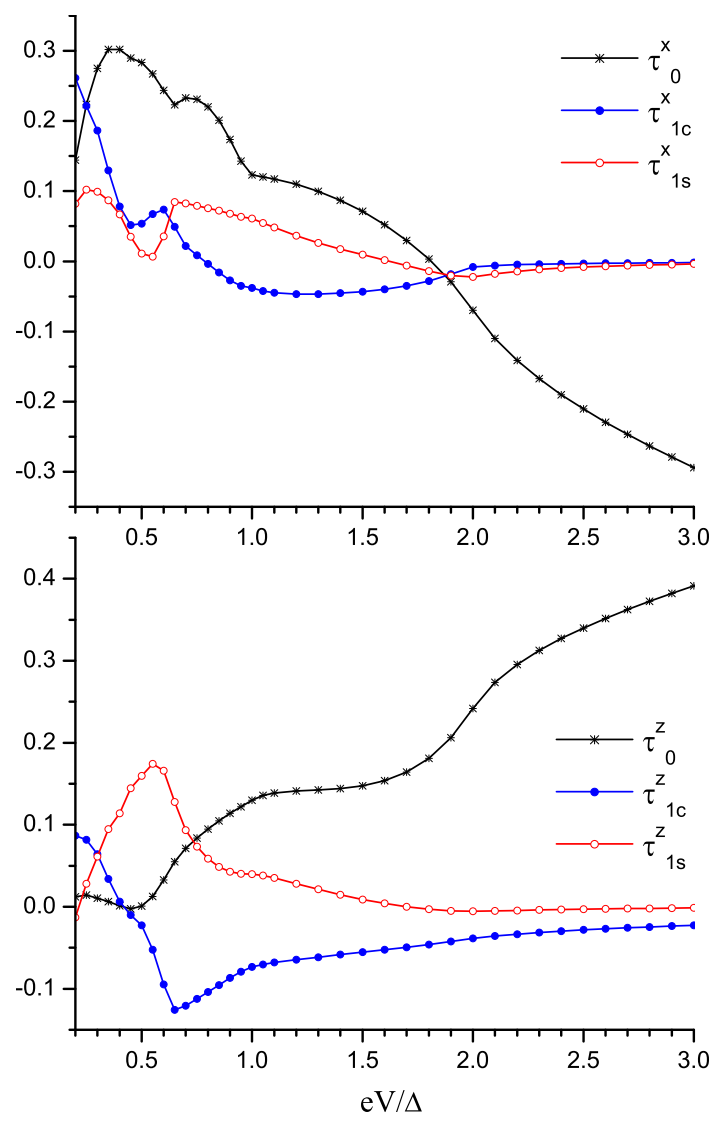

FIGURE 3. Spin-transfer torque on $\mathrm{F}^{b}$ in $x$ (upper panel) and $z$ (lower panel) direction. $D_{\uparrow}=0.95, D_{\downarrow}=0.6, \psi=\pi / 2$.

components of $\vec{\tau}_{b}$, in unit of $N_{f} v_{f} \Delta A \hbar / 2$, as functions of the bias voltage at $T=0$. Notice $\vec{\tau}_{b}$ is perpendicular to $\vec{\mu}_{b}$ and lies in the $x z$ plane $(\psi=\pi / 2)$. An interesting feature of the dc torque along $x$ direction is that it changes sign around voltage $1.8 \Delta / e$. The magnitude of the dc torque becomes linear to $V$ at high voltages. The ac torque becomes vanishingly small compared to the dc torque for $V>2 \Delta$ because the number of subgap Andreev reflections, which constitute the dominant contributions to the ac spin current, is inversely proportional to $V$.

\section{REFERENCES}

1. J. C. Slonczewski, J. Magn. Magn. Mater, 159, L1 (1996).

2. L. Berger, Phys. Rev. B, 54, 9353(1996).

3. M. Tsoi et al., Phys. Rev. Lett., 80, 4281 (1998).

4. S. I. Kiselev et al., Nature, 425, 380 (2003).

5. C. Bell et al., Appl. Phys. Lett., 84, 1153 (2004).

6. X. Waintal and P. W. Brouwer, Phys. Rev. B, 65, 054407 (2002).

7. X. Waintal and P. W. Brouwer, Phys. Rev. B, 63, R220407 (2001). 
8. E. Zhao, T. Löfwander, and J. A. Sauls, Phys. Rev. B, 70, 134510 (2004).

9. J. Rammer and H. Smith, Rev. Mod. Phys., 58, 323 (1986). 